\title{
THERMOSENSITIVE POLY(N-ISOPROPYLACRYLAMIDE)-B-POLYCAPROLACTONE-B-POLY(N- ISOPROPYLACRYLAMIDE) TRIBLOCK COPOLYMERS PREPARED VIA ATOM TRANSFER RADICAL POLYMERIZATION FOR CONTROL OF CELL ADHESION AND DETACHMENT
}

\author{
LIANG LI*, XIAOMING YANG*, FANGJUN LIU, JINGQI SHANG, GUOPING YAN, AND WEN LI
}

\begin{abstract}
Key Laboratory for Green Chemical Process of Ministry of Education, School of Materials Science and Engineering, Wuhan Institute of Technology, Wuhan 430073, College of Chemistry, Chemical Engineering and Materials Sciences, Soochow University, Suzhou 215123, P. R. China
\end{abstract}

(Received: March 9, 2009 - Accepted: July 28, 2009)

\begin{abstract}
Stimuli-responsive polymer materials have potential uses in drug delivery, tissue engineering, bioreactors, and cell-surface adhesion control. Temperatureresponsive surfaces of triblock copolymers of poly(N-isopropylacrylamide)- $b$-polycaprolactone- $b$-poly(N-isopropylacrylamide) (P(NIPAAm)- $b$-PCL- $b$ $\mathrm{P}(\mathrm{NIPAAm})$ ) were fabricated via atom transfer radical polymerization (ATRP). At $37^{\circ} \mathrm{C}$ [above the lower critical solution temperature (LCST) of NIPAAm of $32{ }^{\circ} \mathrm{C}$ ], the seeded cells adhered on the surface of the triblock copolymer, while below the LCST the cells detached from the surface spontaneously. P(NIPAAm) acted as the thermoresponsive segments of the triblock copolymer for control of cell adhesion and detachment. The thermosensitive copolymers are potentially useful as stimuli-responsive adhesion modifiers for cells in biomedical fields.
\end{abstract}

Keywords: block copolymers, ATRP, stimuli-sensitive polymers, cell detachment.

\section{INTRODUCTION}

In the past decade, polymer materials have been widely used in the tissue engineering, drug delivery, bioseparation, chemical separation, water treatment and chemical sensors because of their good resilience, low density, and low cost. ${ }^{1-3}$ However, due to problems including nonselective protein adsorption and nonselective cell adhesion, polymer biomaterials are limited in their applications. Biomaterials with responsive surfaces may solve this problem since their remarkable properties can be controlled or adjusted by some external stimuli, such as temperature, $\mathrm{pH}$, ionic strength, solvents, photoirradiation, electric and magnetic stimulation, etc. ${ }^{4-6}$ This response can be used to regulate the activity of biological tissues, for example, the automatic cell

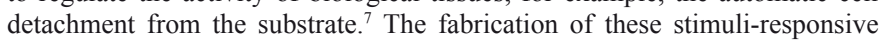
polymer materials is of both scientific and technological interest. ${ }^{8}$

Polycaprolactone (PCL) is a biodegradable and biocompatible linear polyester with good mechanical and thermoplastic properties. ${ }^{9-11}$ Poly $(\mathrm{N}-$ isopropylacrylamide) (P(NIPAAm)) is a well-known thermoresponsive polymer and exhibits a lower critical solution temperature (LCST) of about 32 ${ }^{\circ} \mathrm{C}$ in an aqueous medium. It assumes a random coil structure (hydrophilic state) below the LCST and a collapsed globular structure (hydrophobic state) above the LCST. ${ }^{12-14}$ Various cells can adhere, spread, and proliferate at $37^{\circ} \mathrm{C}$ on the hydrophobic P(NIPAAm)-modified surfaces. However, at temperatures below the LCST of P(NIPAAm), the cultured cells can detach spontaneously from the hydrophilic surfaces without enzymatic digestion. PCL and P(NIPAAm) have been widely used for biomaterials and biomedical applications because of their unique properties..$^{15}$

PCL and related polymers from ring-opening polymerizations usually possess hydroxyl-terminated chains..$^{16,17}$ In the present work, the terminal hydroxyl groups of commercial PCL are reacted with 2-bromoisobutyryl bromide ${ }^{7}$ to produce the 2-bromoisobutyryl-terminated PCL macroinitiators (Br-PCL-Br) for the subsequent atom transfer radical polymerization (ATRP). ATRP is a recently developed controlled radical polymerization method. ${ }^{18}$ $\mathrm{P}(\mathrm{NIPAAm})-b$-PCL- $b$-P(NIPAAm) triblock copolymers are prepared by ATRP from the Br-PCL-Br macroinitiators. The triblock copolymers were investigated by Fourier transform infrared (FTIR), differential scanning calorimetry (DSC), gel permeation chromatography (GPC) and X-ray photoelectron spectroscopy (XPS). The nonbiodegradable P(NIPAAm) blocks in the copolymers acts as the thermoresponsive side chains for the control of cell adhesion and detachment. These stimuli-responsive P(NIPAAm)- $b$-PCL$b$-P(NIPAAm) triblock copolymers have potential applications in biomedical fields.

\section{EXPERIMENTAL}

Materials

Dihydroxyl-terminated polycaprolactone pellets (number of average molecular weight $\left(M_{\mathrm{n}}\right)=41000$ and polydispersity index $\left.(\mathrm{PDI})=1.54\right)$, 2-bromoisobutyryl bromide, N-isopropylacrylamide (NIPAAm), 1,1,4,7,10,10hexamethyltriethylenetetramine (HMTETA), copper(I) bromide (CuBr) and dimethyl sulfoxide (DMSO) were obtained from Aldrich Chemical Co. The solvents were of analytical grade and were used without further purification unless otherwise mentioned.

\section{ATRP synthesis}

For the preparation of ATRP macroinitiator, 2-bromoisobutyrylterminated PCL macroinitiators (Br-PCL-Br, as shown in Fig. 1), PCL powders, triethylamine and methylene chloride were first introduced into a flask with a magnetic stirrer. After the PCL powders had completely dissolved, 2-bromoisobutyryl bromide was added into the flask slowly and the reaction was allowed to proceed at room temperature for $24 \mathrm{~h}$. The resulting Br-PCL-Br macroinitiator was precipitated and washed in excess methanol. The Br-PCL$\mathrm{Br}$ macroinitiator for the subsequent ATRP was dried under reduced pressure.

The P(NIPAAm)- $b$-PCL- $b$-P(NIPAAm) triblock copolymers were synthesized using a molar feed ratio [NIPAAm]/[Br-PCL-Br]/[CuBr] [HMTETA] of 600:1:2:4. NIPAAm, Br-PCL-Br, and HMTETA were introduced into the flask containing $10 \mathrm{~mL}$ DMSO. After Br-PCL-Br and NIPAAm had dissolved completely, the reaction mixture was degassed by bubbling argon through the reaction mixture for $30 \mathrm{~min}$. Then $\mathrm{CuBr}$ was added into the mixture under an argon atmosphere. The reaction mixture was purged with argon for another $10 \mathrm{~min}$. The flask was then sealed and the polymerization was allowed to proceed under continuous stirring at $40{ }^{\circ} \mathrm{C}$ for $3-10 \mathrm{~h}$. The reaction was stopped by diluting with THF. The catalyst complex was removed, bypassing the blue dilute polymer solution through an aluminum oxide column. The P(NIPAAm)- $b$-PCL- $b$-P(NIPAAm) triblock copolymers were precipitated in excess methanol. The crude polymer was purified by reprecipitation twice to remove the reactant residues before dried under reduced pressure.

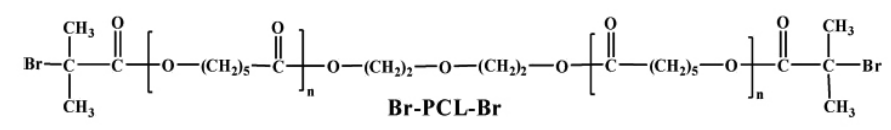

Fig. 1. Molecular structure of the macroinitiator

\section{Characterization}

X-ray photoelectron spectroscopy (XPS) analysis was performed on a Kratos AXIS HSi spectrometer with a monochromotized $\mathrm{Al} \mathrm{K \alpha}$ X-ray source (1486.6 eV photons). All binding energies were referenced to the $\mathrm{C}$ 1s hydrocarbon peak at $284.6 \mathrm{eV}$. Surface elemental stoichiometries were determined from the sensitivity factors-corrected spectral area ratios and were reliable to within $\pm 5 \%$. Fourier transform infrared (FTIR) spectra were recorded on a Bruker IFS66V spectrometer. The spectra were collected by cumulating 64 scans. Before measuring the lower critical solution temperature (LCST) of the P(NIPAAm) blocks in the triblock copolymers, all samples were immersed in deionized water at room temperature for $48 \mathrm{~h}$ to reach the equilibrium state. Then the samples were placed in individual hermetic sample pans and sealed. LCST was determined by differential scanning calorimetry 
(TA 2920 Modulated DSC, TA Instruments) with a heating rate of $3{ }^{\circ} \mathrm{C} / \mathrm{min}$ under a nitrogen flow rate of $40 \mathrm{~mL} / \mathrm{min}$, using deionized water as the reference. Gel permeation chromatography (GPC) measurements were performed on a Waters GPC system equipped with a set of Waters Styragel columns, a Waters2487 dual $\lambda$ absorbance detector, and a Waters-2414 refractive index detector Monodispersed polystyrene standards were used to generate the calibration curve. THF of HPLC grade was used as the diluent at a flow rate of $1.0 \mathrm{~mL} /$ min. 1H NMR spectra were measured by an INOVA400 nuclear magnetic resonance (NMR) spectrometer using $\mathrm{CDCl}_{3}$ as the solvent.

\section{Cell culture on the copolymer surfaces}

Before the experiments of the cell adhesion and detachment for the triblock copolymers, the membrane of the copolymer was prepared by the well-known phase inversion technique from the $15 \mathrm{wt} \%$ dioxane solution of the P(NIPAAm)- $b$-PCL- $b$-P(NIPAAm) triblock copolymer in water. Dry membranes with a thickness of about $30 \mu \mathrm{m}$ were obtained after pumping under reduced pressure. For the cell culture on the membranes of the triblock copolymer, the membranes were washed twice with phosphate-buffered saline (PBS) solution and then sterilized for about $1 \mathrm{~h}$ by UV irradiation, prior to being placed into the wells of a 12-well culture plate. 3 T3 fibroblasts (ATCC, Passage 27) were seeded into the wells at a density of $1 \times 10^{4}$ cells $/$ well and incubated (in 1ml Dulbecco's modified eagle medium (DMEM) supplemented with $10 \%$ fetal bovine serum, $1 \mathrm{mM}$ L-glutamine, and 100 units $/ \mathrm{ml}$ penicillin at $37{ }^{\circ} \mathrm{C}$ ) for 2 days under a humidified $5 \% \mathrm{CO}_{2}$ atmosphere, and then washed twice in $37{ }^{\circ} \mathrm{C}$ PBS solution to remove the loosely attached cell. Cell fixation with $4 \%$ glutaraldehyde for $2 \mathrm{~h}$ and dehydration in a series of ethanol solutions $(50-100 \%)$ were carried out. Cell adhesion and cell detachment were carried out at $37{ }^{\circ} \mathrm{C}$ (above the LCST of P(NIPAAm)) and at $20^{\circ} \mathrm{C}$ (below the LCST of P(NIPAAm), respectively. The membrane surfaces with immobilized cells were imaged by an Olympus BX51M optical microscope (Olympus America Inc.). The cell number on each membrane was counted on printed photographs from three or more samples and averaged. ${ }^{19}$

\section{RESULTS AND DISCUSSION}

ATRP synthesis of the P(NIPAAm)-b-PCL-b-P(NIPAAm) triblock copolymers

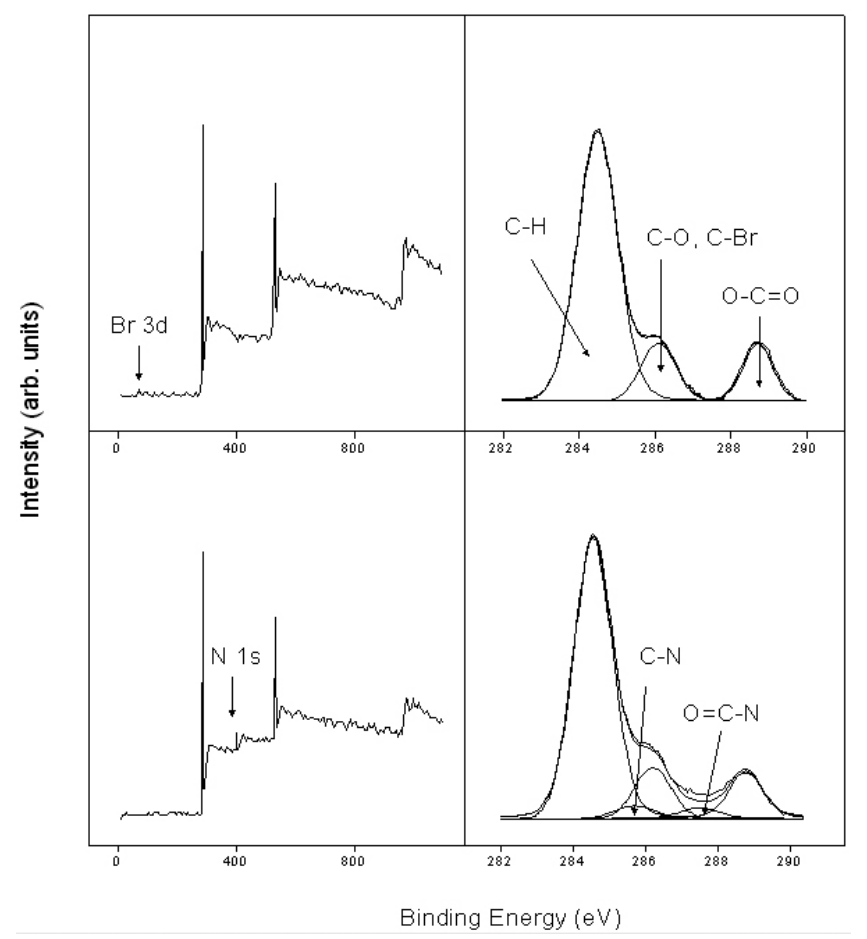

Fig. 2. XPS wide scan and C 1s core-level spectra of the Br-PCL-Br macroinitiator $(\mathrm{a}, \mathrm{b})$ and the triblock copolymer2 $(\mathrm{c}, \mathrm{d})$
The Br-PCL-Br macroinitiator for ATRP was prepared via reaction of the terminal hydroxyl groups of PCL with 2-bromoisobutyrl bromide. Then the P(NIPAAm)- $b$-PCL- $b$-P(NIPAAm) triblock copolymer was synthesized via ATRP of NIPAAm from the Br-PCL-Br macroinitiator. The chemical composition of the copolymers was determined by XPS. Fig. 2 shows the XPS wide scan and C 1s core-level spectra of the Br-PCL-Br macroinitiator and the triblock copolymer2 (from $7 \mathrm{~h}$ of ATRP).

In the wide scan spectrum of the Br-PCL-Br macroinitiator, a weak $\mathrm{Br} 3 \mathrm{~d}$ signal at the binding energy (BE) of about $70 \mathrm{eV}$, characteristic of covalently bonded bromine, ${ }^{20}$ has been observed. The $\mathrm{C} 1 \mathrm{~s}$ core-level spectrum of the $\mathrm{Br}$ PCL-Br macroinitiator (Fig. 2b) can be curve-fitted into three peak components with BEs at about 284.6, 286.2, and $288.7 \mathrm{eV}$, attributable to the C-H, C-O/C$\mathrm{Br}$, and $\mathrm{O}=\mathrm{C}-\mathrm{O}$ species, respectively. ${ }^{20}$ Compared with the wide scan spectrum of the Br-PCL-Br macroinitiator, a relatively strong $\mathrm{N}$ 1s signal at the BE of about $399 \mathrm{eV}$ has appeared in the wide scan spectra of triblock copolymers (Fig. 2c). As shown in Fig. 2d, the corresponding C 1s core-level spectra of the copolymers can be curve-fitted into five peak components with BEs at about 284.6, 285.7, 286.2, 287.4, and $288.7 \mathrm{eV}$, attributable to the C-H, C-N, C-O, $\mathrm{O}=\mathrm{C}-\mathrm{N}$, and $\mathrm{O}=\mathrm{C}-\mathrm{O}$ species, respectively. ${ }^{20}$ The $\mathrm{C}-\mathrm{N}$ and $\mathrm{O}=\mathrm{C}-\mathrm{N}$ species are associated with the P(NIPAAm) blocks. From the XPS derived $[\mathrm{N}] /[\mathrm{C}]$ ratio, the P(NIPAAm) content in each block polymer can also be estimated. Table 1 summarizes the results of the copolymers as a function of polymerization time. Triblock copolymers with different contents of the P(NIPAAm) block were synthesized by varying the ATRP time. With the increase in reaction time from 3 to $10 \mathrm{~h}$, the $M_{\mathrm{n}}$ of the copolymer increases from $4.6 \times 10^{4}$ to $5.6 \times 10^{4} \mathrm{~g} / \mathrm{mol}$ and the P(NIPAAm) content increases accordingly from 11.4 to $27.0 \mathrm{~mol} \%$. In addition, PDIs of the triblock copolymers are comparable to that of the starting $\mathrm{Br}-\mathrm{PCL}-\mathrm{Br}$, which indicates that the ATRP of NIPAAm from Br-PCL-Br is controlled.

Table 1 Characterization of the P(NIPAAm)- $b$-PCL- $b$-P(NIPAAm) triblock copolymers

\begin{tabular}{|c|c|c|c|c|c|}
\hline sample & $\begin{array}{c}\text { reaction } \\
\text { time }\end{array}$ & $\begin{array}{c}M_{\mathrm{n}}(\mathrm{g} / \\
\mathrm{mol}^{b}\end{array}$ & $\mathrm{PDI}^{b}$ & {$[\mathrm{~N}] /[\mathrm{C}]^{c}$} & $\begin{array}{c}\text { P(NIPAAm) } \\
\text { content } \\
(\mathrm{mol} \%)^{d}\end{array}$ \\
\hline PCL & & 41000 & 1.54 & & \\
\hline copolymer1 $^{a}$ & 3 & 46000 & 1.50 & 0.019 & $11.4^{e}$ \\
\hline copolymer2 $^{a}$ & 7 & 52000 & 1.48 & 0.033 & $19.8^{e}$ \\
\hline copolymer3 $^{a}$ & 10 & 55000 & 1.47 & 0.045 & $27.0^{e}$ \\
\hline
\end{tabular}

${ }^{a}$ Synthesized using a molar feed ratio [monomer]/[Br-PCL-Br)]/[CuBr] $/$ [HMTETA] of 600:1:2:4. ${ }^{b}$ Determined from GPC results. ${ }^{c}$ Determined from XPS N $1 \mathrm{~s}$ and C $1 \mathrm{~s}$ core-level spectral area ratio. ${ }^{d}$ Calculated from $n_{\text {[NIPAAm] }}$ $\left(n_{\text {[NIPAAm] }}+n_{\text {[CL] }}\right) .{ }^{e}$ Determined from $[\mathrm{N}] /[\mathrm{C}]$ ratio, where $[\mathrm{N}] /[\mathrm{C}] \approx n_{[\mathrm{NIPAAm}]} /$ $\left(6 n_{[\mathrm{NIPAAm}]}+6 n_{[\mathrm{CL}]}\right)$

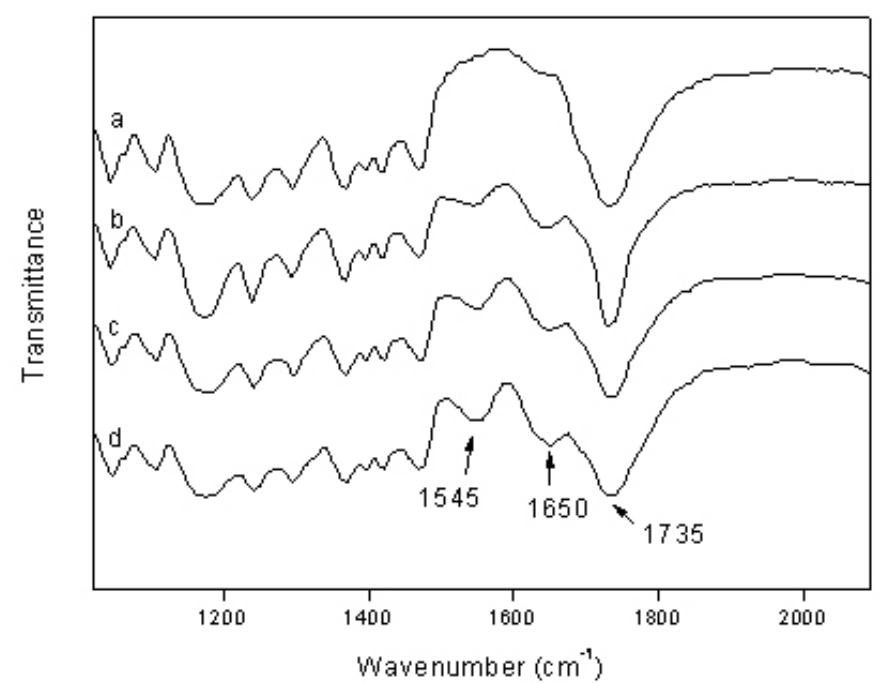

Fig. 3. FTIR spectra of the Br-PCL-Br macroinitiator (a), the triblock copolymer1 (b), copolymer2 (c) and copolymer3 (d). 
FTIR spectroscopy was also used to characterize the chemical structure of the polymers. Fig. 3 shows the FTIR spectra of the Br-PCL-Br macroinitiator, the triblock copolymer1, copolymer2 and copolymer3. In all the FTIR spectra, the characteristic band at about $1735 \mathrm{~cm}^{-1}$ which is assigned to the $v(\mathrm{O}=\mathrm{C}-\mathrm{O})$ vibration, is associated with PCL. Its relative intensity decreases with the increase in P(NIPAAm) content in the P(NIPAAm)-b-PCL-b-P(NIPAAm) triblock copolymers. The typical amide absorption bands of P(NIPAAm) at about $1650(\mathrm{v}(\mathrm{O}=\mathrm{C}-\mathrm{NH})$ vibration $)$ and $1545 \mathrm{~cm}^{-1}(\mathrm{v}(\mathrm{N}-\mathrm{H})$ vibration $)$ are observed only in the FTIR spectra of the P(NIPAAm)-b-PCL- $b$-P(NIPAAm) triblock copolymers. The relative intensities of both peaks at 1650 and 1545 $\mathrm{cm}^{-1}$ increase significantly with the increase in P(NIPAAm) content in the triblock copolymers. The above FTIR results are thus consistent with the XPS results.

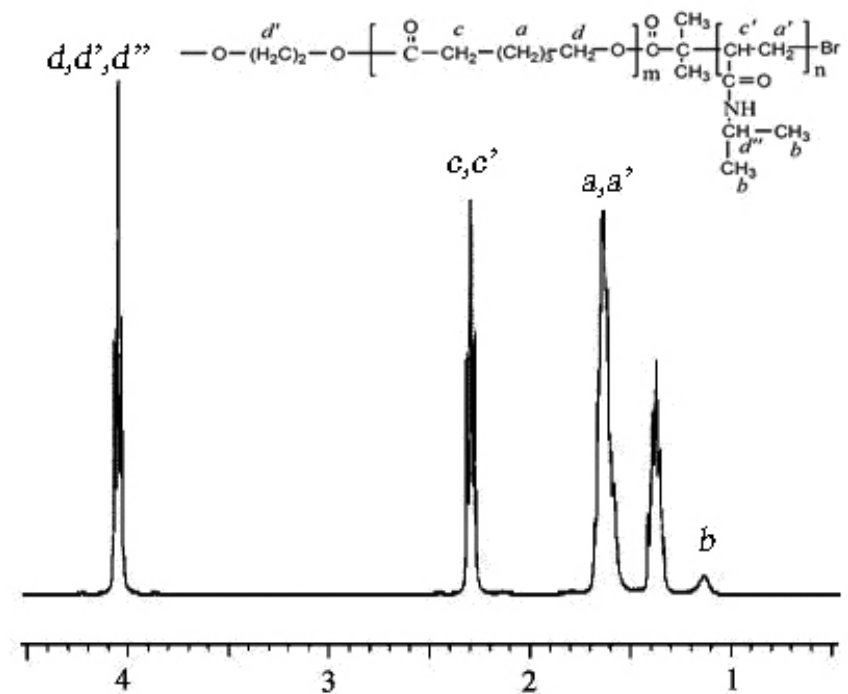

Fig. 4. 1H NMR spectra of the triblock copolymer1 in $\mathrm{CCl}_{3} \mathrm{D}$.

The chemical structures of the triblock copolymer were characterized by $1 \mathrm{H}$ NMR spectroscopy. The chemical shifts at $\delta=1.13 \mathrm{ppm}$ are mainly associated with the methyl protons (b, $\left.\mathrm{CH}(\mathrm{NH})-\mathrm{CH}_{3}\right)$ of the P(NIPAAm) blocks. The chemical shifts in the region of 1.3-1.8 ppm are attributable to the methylene protons ( $a, \mathrm{CH}_{2}-\mathrm{CH}_{2}$ and $\left.a^{\prime}, \mathrm{CH}-\mathrm{CH}_{2}\right)$. The chemical shifts at $\delta$ ) 2.2-2.4 ppm are associated with the methylene $\left(c, \mathrm{CH}_{2}-\mathrm{C}=\mathrm{O}\right)$ and methylidyne $\left(c^{\prime}, \mathrm{CH}-\mathrm{C}=\mathrm{O}\right)$ protons adjacent to the carbonyl group. The chemical shifts at $\delta=4.0-4.25 \mathrm{ppm}$ correspond to the methylene protons $\left(d, d^{\prime}, \mathrm{CH}-\mathrm{O}-\mathrm{C}=\mathrm{O}\right)$

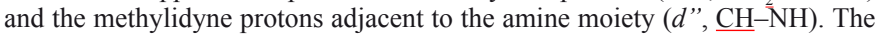
results are in good agreement with those obtained from XPS.

The lower critical solution temperature (LCST) of the P(NIPAAm) blocks in the triblock copolymers was determined by DSC. DSC thermograms of the Br-PCL-Br macroinitiator, the triblock copolymer1, copolymer2 and copolymer3 are shown in Fig. 5. The LCST of thermo-responsive P(NIPAAm) is the consequence of hydrophobic (associated with the isopropyl groups) and hydrophilic (associated with the amide moiety in the pendent groups) interactions of P(NIPAAm) in aqueous enviroment. The temperature at the minimum point of the endotherm is referred to as the LCST of each sample. As expected, LCST can not be observed for PCL. On the other hand, the P(NIPAAm)- $b$-PCL- $b$-P(NIPAAm) triblock copolymer1, copolymer2 and copolymer3 exhibit LCSTs at about $30.8,31.0$ and $31.2{ }^{\circ} \mathrm{C}$ (near that of the $\mathrm{P}(\mathrm{NIPAAm})$ homopolymer at about $32{ }^{\circ} \mathrm{C}$ ), respectively, indicating that the PCL segments do not have a significant effect on the LCST of the P(NIPAAm) blocks in an aqueous medium. Zhang et al. reported that incorporation of hydrophobic moieties in the bulk may decrease the LCST of P(NIPAAm). ${ }^{21}$ When a hydrophobic component was incorporated into P(NIPAAm), the hydrophilic-hydrophobic balance will shift toward a more hydrophobic nature and the LCST will shift to a lower temperature. Below the LCST, the P(NIPAAm) blocks in the triblock copolymers are solvated and remain fully extended as a hydrophilic phase in an aqueous medium, while the PLC blocks associate hydrophobically and precipitate out from the aqueous medium. ${ }^{12}$ The strong repulsion between the P(NIPAAm) blocks and the PCL blocks will lead to phase separation in an aqueous medium. The phase separation probably has limited the interaction of the hydrophobic PCL blocks with the P(NIPAAm) blocks. Thus, the LCST of the P(NIPAAm) blocks is not significantly affected by the hydrophobic PCL blocks.

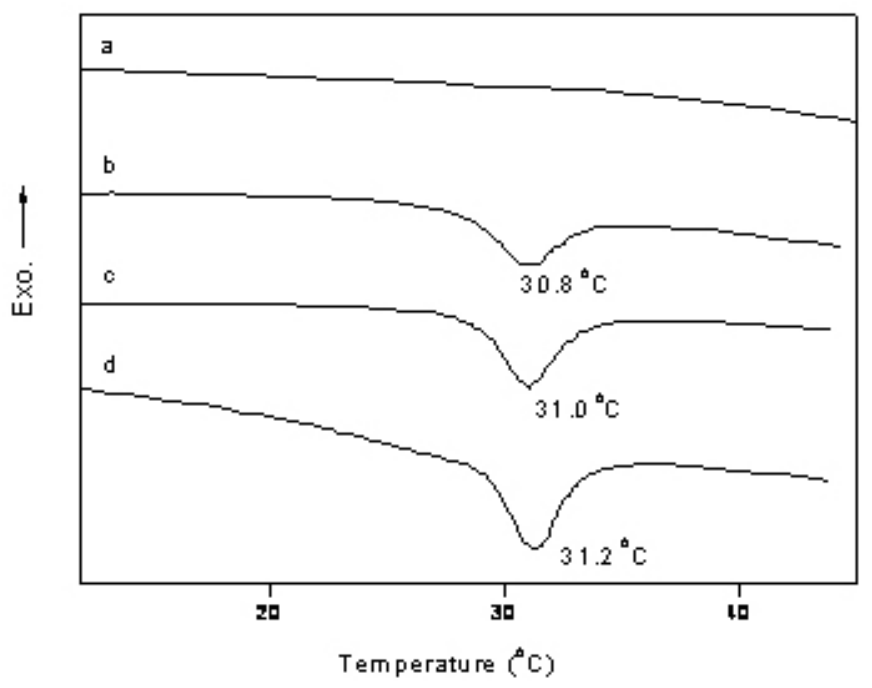

Fig. 5. DSC thermograms of the Br-PCL-Br macroinitiator (a), the triblock copolymer1 (b), copolymer2 (c) and copolymer3 (d).

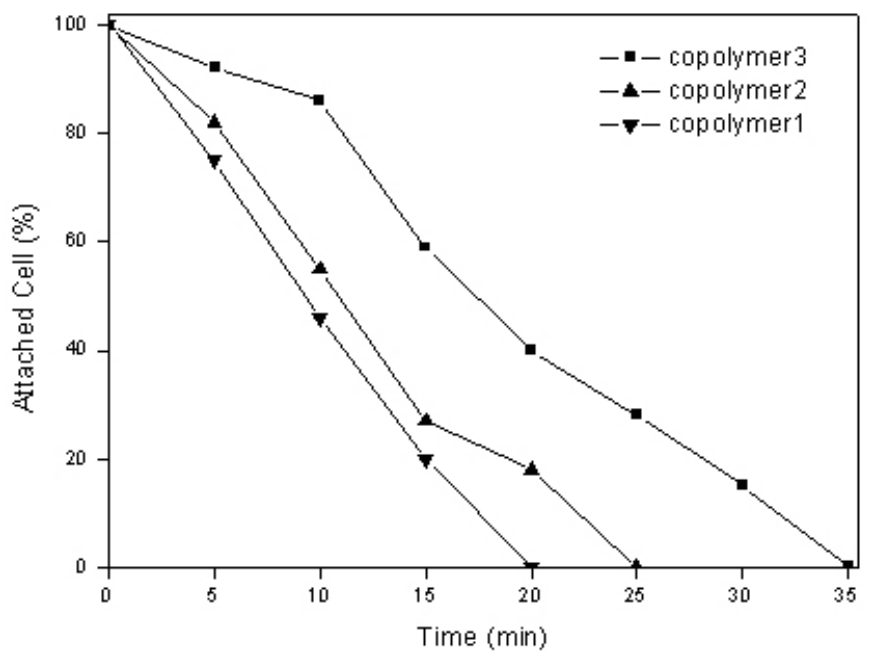

Fig. 6. Time-dependent cell detachment from the surfaces of the triblock copolymer membranes upon reducing the culture temperature to $20^{\circ} \mathrm{C}$.

Cell adhesion and detachment characteristics of the triblock copolymer surfaces

P(NIPAAm) exhibits a LCST of about $32^{\circ} \mathrm{C}$ in an aqueous medium. On the surfaces containing P(NIPAAm), cells can adhere, spread, and proliferate at 37 ${ }^{\circ} \mathrm{C}$. However, at temperatures below the LCST of P(NIPAAm), the cultured cells detach spontaneously from the hydrophilic surfaces in the absence of enzymatic digestion. ${ }^{22,23}$ In the present work, temperature-dependent cell detachment from the surface of the triblock copolymers is demonstrated. Before the experiments of the cell adhesion and detachment for the triblock copolymers, the membrane of the copolymer was prepared by the well-known phase inversion technique from the $15 \mathrm{wt} \%$ dioxane solution of the P(NIPAAm)- $b$-PCL- $b$-P(NIPAAm) triblock copolymer in water at a predetermined temperature. The cells can adhere and grow to some extent on the surfaces at $37{ }^{\circ} \mathrm{C}$. At $37{ }^{\circ} \mathrm{C}$, the P(NIPAAm) segments of these surfaces associate hydrophobically and collapse into globular 
structures which can support cell attachment, spread, and proliferation. The above results suggest that the thermoresponsive copolymer do not restrain cell attachment at $37^{\circ} \mathrm{C}$. Cell detachment from the membrane surfaces was studied by lowering the incubation temperature. Significant amount of cells have detached from the surfaces after the low-temperature treatment, as shown in Fig. 6. When the culture temperature was lowered to $20^{\circ} \mathrm{C}$, the P(NIPAAm) chains on the surface become hydrated below the LCST, producing an expanded, swollen, and hydrophilic surface. This change in surface property weakens cellular adhesion, resulting in spontaneous cell detachment from the extended P(NIPAAm) block. Within $15 \mathrm{~min}$, about $80 \%, 75 \%$ and $40 \%$ of the adhered cells have detached, respectively, from the copolymer1 surface, copolymer2 surface and copolymer3 surface. For complete cell detachment, about 20, 25 and 35 min were required for the copolymer1 surface, copolymer2 surface and copolymer3 surface, respectively. The rate of cell detachment from the copolymer1 surface is faster than that from the other surfaces. The phenomenon probably arises from the longer time required to hydrate the P(NIPAAm) in the copolymers containing more P(NIPAAm) block.

\section{CONCLUSIONS}

Thermosensitive P(NIPAAm)- $b$-PCL- $b$-P(NIPAAm) triblock copolymers are prepared via atom transfer radical polymerization from the Br-PCL-Br macroinitiator. The resultant P(NIPAAm) blocks act as the thermoresponsive segments of the copolymer for the control of cell adhesion and detachment. At $37^{\circ} \mathrm{C}$ (above the LCST of P(NIPAAm) of $32^{\circ} \mathrm{C}$ ), the hydrophobic P(NIPAAm) segments associated hydrophobically to form a collapsed globular structure which supported cell attachment, spread, and proliferation. When the culture temperature was lowered to $20^{\circ} \mathrm{C}$, the hydration of P(NIPAAm) blocks results in spontaneous cell detachment. The thermosensitive triblock copolymers are potentially useful for the fabrication of bio- and molecular sensors.

\section{ACKNOWLEDGEMENTS}

The work is supported by Educational Bureau of Hubei Province (Q20091508), Scientific Research Key Project of Ministry of Education of China (209081), Key Project in Science \& Technology Innovation Cultivation Program of Soochow University and National Natural Science Foundation of China.

\section{REFERENCES}

1. A.V. Reis, M.R. Guilherme, O.A. Cavalcanti, A.F. Rubira, E.C. Muniz, Polymer 47, 2023, (2006).

2. S.J. Lee, Y. Bae, K. Kataoka, D. Kim, D.S. Lee, S.C. Kim, Polym. J. 40, $171,(2008)$.
3. T. Nguyen, B. Farrugia, T.P. Davis, C. Barner-Kowollik, M.H. Stenzel, J. Polym. Sci. Part A: Polym. Chem. 45, 3226, (2007).

4. M. Karg, I. Pastoriza-Santos, B. Rodriguez-Gonzalez, R. Klitzing, S. Wellert, T. Hellweg, Langmuir 24, 6300, (2008).

5. K.V. Bernaerts, C.A. Fustin, C. Bomal-Dhaese, J.F. Gohy, J.C. Martins, F.E.Du Prez, Macromolecules 41, 2593, (2008).

6. S.A. Pooley, B.L. Rivas, A.L. Carcamo, G.D.C.Pizarro, J. Chil. Chem. Soc. 53, 1483, (2008).

7. F.J. Xu, S.P. Zhong, L.Y.L. Yung, E.T. Kang, K.G. Neoh, Biomacromolecules 5, 2392, (2004).

8. S.K. Ahn, R.M. Kasi, S.C. Kim, N. Sharma, Y.X. Zhou, Soft Mater. 4, $1151,(2008)$.

9. K. Filipczak, M. Wozniak, P. Ulanski, L. Olah, G. Przybytniak, R.M. Olkowski, M. Lewandowska-Szumiel, J.M. Rosiak, Macromol. Biosci. 6, 261, (2006).

10. B.J. Papenburg, L. Vogelaar, L.A.M. Bolhuis-Versteeg, R.G.H. Lammertink, D. Stamatialis, M. Wessling, Biomaterials 28, 1998, (2007).

11. K. Uto, K. Yamamoto, S. Hirase, T. Aoyagi, J. Controlled Release 110, 408, (2006).

12. F.J. Xu, J. Li, J. Yuan, Z.X. Zhang, E.T. Kang, K.G. Neoh, Biomacromolecules 9, 331, (2008).

13. N. Ishida, S. Biggs, Macromolecules 40, 9045, (2007).

14. A. Harada, K. Johnin, A. Kawamura, K. Kono, J. Polym. Sci. Part A: Polym. Chem. 45, 5942, (2007).

15. H.V. Recum, T. Okano, S.W. Kim, J. Controlled Release 55, 121, (1998).

16. G. Wang, Y. Shi, Z. Fu, W. Yang, Q. Huang, Y. Zhang, Polymer 46, 10601, (2005).

17. M. Schappacher, A. Soum, S.M. Guillaume, Biomacromolecules 7, 1373, (2006).

18. S. Edmondson, V.L. Osborne, W.T.S. Huck, Chem. Soc. Rev. 35, 14, (2004).

19. M. Ebara, M. Yamato, M. Hirose, T. Aoyagi, A. Kikuchi, K. Sakai, T. Okano, Biomacromolecules, 4, 344, (2003).

20. J.F. Moulder, W.F. Stickle, P.E. Sobol and K.D. Bomben in X-ray Photoelectron Spectroscopy, J. Chastain eds. Perkin-Elmer, Eden Prairie, MN, 1992

21. X.Z. Zhang, Y.Y. Yang, T.S. Chung, K.X. Ma, Langmuir 17, 6094, (2001).

22. J.H. Cho, S.H. Kim, K.D. Park, M.C. Jung, W.I. Yang, S.W. Han, Biomaterials 25, 5743, (2004).

23. D. Schmaljohann, J. Oswald, B. Jørgensen, M. Nitschke, D. Beyerlein, C. Werner, Biomacromolecules 4, 1733, (2003). 